\title{
Time-Memory Trade-offs for Index Calculus in Genus 3
}

\author{
Kim Laine ${ }^{1}$ and Kristin Lauter ${ }^{2}$ \\ 1 University of California, Berkeley \\ laine@math. berkeley .edu \\ 2 Microsoft Research, Redmond \\ klauter@microsoft.com
}

\begin{abstract}
In this paper, we present a variant of Diem's $\widetilde{O}(q)$ index calculus algorithm to attack the discrete logarithm problem (DLP) in Jacobians of genus 3 non-hyperelliptic curves over a finite field $\mathbb{F}_{q}$. We implement this new variant in $\mathrm{C}++$ and study the complexity in both theory and practice, making the logarithmic factors and constants hidden in the $\widetilde{O}$-notation precise. Our variant improves the computational complexity at the cost of a moderate increase in memory consumption, but we also improve the computational complexity even when we limit the memory usage to that of Diem's original algorithm. Finally, we examine how parallelization can help to reduce both the memory cost per computer and the running time for our algorithms.
\end{abstract}

Keywords: discrete logarithm problem, index calculus, double large prime, higher genus, genus 3, non-hyperelliptic curve, quartic curve, plane curve, time-memory trade-off

\section{Introduction}

The discrete logarithm problem (DLP) in the Jacobian of a genus $g$ curve with $g>1$ over a finite field $\mathbb{F}_{q}$ was proposed for use in public key cryptosystems by Koblitz [Kob]. The potential advantage over elliptic curve cryptosystems comes from the fact that $\# \operatorname{Jac}_{\mathcal{C}}\left(\mathbb{F}_{q}\right)=q^{g}+O\left(q^{\frac{2 g-1}{2}}\right)$. So the underlying finite field can be smaller, while still yielding a group of the same size with potentially the same security level. In genus 2 there are no known attacks faster than the generic square root attacks, and the smaller field size and alternate models for the group make the arithmetic very efficient, even efficient enough to be competitive with elliptic curve cryptography ( $\mathrm{BCHL}$, BCLS] $)$.

However for curves of very large genus, subexponential index calculus attacks were discovered by Adleman, DeMarrais, and Huang [ADH], and improved by Gaudry, Enge, and others Gau, Eng, EG, VJS. For small genus but still with $g>3$, the expected security was drastically reduced by attacks, which, although exponential, were so much better than square-root attacks so as to render the complexity/security trade-off unacceptable (e.g. Gau, The, GTTD, Nag]). The case of genus 3 is less clear. The hyperelliptic locus of genus 3 curves is of codimension 1 so almost all genus 3 curves are non-hyperelliptic. The non-hyperelliptic curves are smooth plane quartics due to the canonical embedding. The rest are hyperelliptic of higher degree. A double large prime index calculus algorithm (GTTD) in the hyperelliptic case reduces the complexity of the DLP from $\widetilde{O}\left(q^{3 / 2}\right)$ using Pollard rho to $\widetilde{O}\left(q^{4 / 3}\right)$, where $\widetilde{O}$ hides both constants and logarithmic factors, but this does not seem to be efficient enough to rule out the use of genus 3 hyperelliptic curves in cryptography. On non-hyperelliptic curves the simpler geometry can be exploited to yield more efficient attacks. Diem has been developing index calculus algorithms on Jacobians of low-degree plane curves [Die, Die2, in particular the case of non-hyperelliptic genus $3[\mathrm{DT}$. These more efficient algorithms show that the complexity of solving the DLP on a non-hyperelliptic genus 3 Jacobian is at most $\widetilde{O}(q)$.

But the $\widetilde{O}$-notation is often quite misleading, since the hidden constants and logarithmic factors can be significant for field sizes of practical interest. In this paper, we develop a variant of Diem's algorithm which improves the computational complexity at the cost of a moderate increase in memory consumption. We implemented this new variant in $\mathrm{C}++$ and studied the complexity in both theory and practice, making the logarithmic factors and constants hidden in the $\widetilde{O}$-notation precise. We found clear estimates for the security of non-hyperelliptic genus 3 Jacobians which have to be considered when designing genus 3 hyperelliptic cryptosystems, due to possible isogeny attacks on hyperelliptic Jacobians as given in Smi].

Perhaps the biggest limitation of all algorithms of this type is that they require massive amounts of memory. We study a variant of the algorithm where memory consumption is restricted and observe that the maximum improvement in the running time (compared to Diem's algorithm) can 
nearly be achieved with a much smaller than expected increase in memory use (again compared to Diem's algorithm). We also consider a scenario where memory use is much more severely restricted, in which case we compare our running time to that of Pollard rho. Finally we look at how parallelization can help to reduce both the memory cost per computer and the running time.

\subsection{Overview}

In contrast to ordinary index calculus where only relations between factor base elements are considered, in double large prime index calculus methods also relations with up to two non-factor base elements (large primes) are allowed. The data is structured as a graph (or a tree) which is eventually used to cancel out the large primes to yield relations involving only factor base elements, which are then used in the linear algebra stage of the index calculus algorithm. Diem observed that in the case of plane quartic curves generating relations is particularly easy. One can pass a line through pairs of points on the curve and this line then intersects the curve (over an algebraically closed field) in another 2 points. This gives a relation which holds in the group (the Jacobian of the curve) and can be used in index calculus. Using a fixed initial factor base of size $\widetilde{O}\left(q^{1 / 2}\right)$, each pair of factor base elements yields another two points on the line, and if they are rational over the base field and if one of them is in the factor base or the tree already and the other is not, then the other point may be added to the tree and tagged with this relation.

Once the tree is built and has size $q^{3 / 4}$, a relation-finding stage commences. The goal of this stage of index calculus is to produce relations involving only factor base elements, called full relations. Remaining unused pairs of factor base elements generate additional pairs of points, which, if they are already in the tree, can be used to trace down to obtain full relations, involving only factor base elements. The version of Diem's algorithm from Die2 is described precisely below in Section 2

New variant. A rough description of our new algorithm is as follows. A precise description is given in the algorithm presented in Section 3 and total time and storage cost is given in Section 4 We start with an initial factor base of much smaller size, $\widetilde{O}\left(q^{1 / 8}\right)$, which we then expand into a full factor base of size $\widetilde{O}\left(q^{1 / 2}\right)$ as follows.

At the base vertices construction stage, we take all pairs of elements of the initial factor base, find the other two points on the curve which lie on the line intersecting the factor base points, and (if they are rational) add one of them to the factor base and one to the graph and call it a base vertex, connected to the base (special node) of the graph. This stage is very efficient because it simultaneously builds the factor base and the graph, with very high probability at each step because it builds the graph as long as not both are in the graph already, and the graph is very small to begin with.

The next step is to build triangle relations. We take all pairs of base vertices, find the other two points on the line and the curve, and add one to the factor base, and one to the graph. The one added to the graph is called a top triangle vertex. At the end of this stage, the graph consists of many triangles, the bottom two triangle vertices are connected directly to the factor base (the special node), and the top vertex is connected to the base triangle vertices (with a relation involving one element of the factor base). At this point, the factor base consists of roughly $q^{1 / 2}$ elements, roughly the same as the size of the graph.

The next stage continues graph building while also starting to collect full relations. Essentially we will build tree branches on top of the top triangle vertices, while also collecting relations. That way we can make use of relations both when the new points are in the graph and when one of them is not. We only discard a relation if both of the new points are not in the graph. This stage terminates when enough full relations have been generated. In Theorem 2 we show that if the size of the factor base is roughly $4 \lambda^{4} q^{1 / 2}$, the graph grows to size $4 \lambda^{2} q^{3 / 4}$, where $\lambda$ satisfies $\lambda \exp \left(4 \lambda^{8}\right)=q^{1 / 8}$, and the algorithm can be expected to terminate successfully.

For the linear algebra step, we don't make any specific changes, but we will show that our average row weight is a constant factor less than that in Diem's algorithm (Lemma 2, which leads to an improvement in running time of the linear algebra stage.

Note that for both our algorithm and Diem's, the entire graph building and relation finding stages do not use the (expensive) group operation on the Jacobian of the plane quartic curve. Instead each step requires only finding the other two points of intersection of a line with the curve. The complexity of this operation is logarithmic in $q$ and in the characteristic 2 case it is particularly simple, as we explain below. The running time of our algorithm presented here refers to the characteristic 2 case. 
Performance. To understand the overall performance of this type of attack on the discrete logarithm problem, we must estimate the time and memory costs for both the relation collection stage and the linear algebra stage. To maximize efficiency, these algorithms are generally designed so that the time and memory costs in the two stages are relatively balanced. In Table 1 , we estimate the time and memory costs for the relation collection stage of our algorithm for field sizes of interest, $2^{30} \leq q \leq 2^{240}$, based on the theoretical results from Theorem 2. Time complexity is measured in terms of counting the number of field multiplications required, and based on rough estimates of the parameters for our algorithm where $q$ is in the range of $2^{70} \leq q \leq 2^{120}$ (see Remark 1), we estimate the running time to be

$$
\approx 1.23123 \cdot \log _{2}^{2}(q) \cdot q
$$

in the binary case. Table 1 shows for example, that to achieve a 128-bit security level measured in terms of time complexity, $q$ should be at least 115 bits. This ignores, however, the memory requirements for these algorithms, which is the size of the graph, $\widetilde{O}\left(q^{3 / 4}\right)$ for both. Table 2 gives experimental results, for small $q$, and shows that in practice the performance of our implementation of our algorithm matches very well with the theoretical predictions.

Comparison with Diem's algorithm. Our algorithm gives a constant factor improvement over Diem's algorithm in two different ways. First, the time required for the relation search step is dominated by the graph-building and relation-finding step of our algorithm, and this is proportional to the square of the size of the factor base. In Lemma 2 we show that the squared ratio of the size of Diem's factor base to the size of our factor base approaches 3 (from above) as $q$ increases. In addition, the graph that we build is wider and not as deep as Diem's graph, which leads to an improvement in the row weight of our vectors for the linear algebra stage. In Lemma 2 we show that we improve the linear algebra stage by a factor which approaches 9 asymptotically as $q$ increases. We obtain these improvements at the cost of increasing the size of the graph used in relation collection, which increases the memory requirements of the algorithm. Our graph size is roughly $4 \lambda^{2} q^{3 / 4}$ as compared to Diem's $q^{3 / 4}$. Table 1 shows values for $\lambda$ which are very close to 1 for all $q$ in the range considered.

Time-memory trade-offs. It is clear from Table 1 that the memory cost is a huge bottleneck in the algorithm. To help with this, we study another version of our algorithm, which stops graph building when the graph has reached a certain size and continues only with relation search. A key observation is that one can restrict the memory cost by a significant constant factor without changing the computational complexity too much. These results can be seen in Figure 1 and Figure 2, It is worth pointing out that even if we restrict our graph size to be the same as Diem's, we still get an improvement in the running time. At another extreme, we study how the complexity behaves when the graph size is restricted to be much smaller than in the unrestricted case and compare the running time to that of Pollard rho.

\section{Diem's Index Calculus}

There are several variants of Diem's index calculus for low degree plane curves. We restrict to the case of non-hyperelliptic genus 3 curves over finite fields embedded as smooth plane quartics using the canonical embedding. These are all double large prime index calculus algorithms where elements of the group are decomposed into sums of factor base elements and at most two nonfactor base elements (large primes). The large primes are then eliminated using various methods to produce relations consisting only of factor base elements.

Let $C$ be a non-hyperelliptic curve of genus 3 over a finite field $\mathbb{F}_{q}$. Using the canonical embedding it can be realized as a smooth plane quartic. Let $P_{0}$ be an $\mathbb{F}_{q}$-rational point on $\mathcal{C}$. The algorithms find $x$ in the DLP

$$
D_{2}-3\left[P_{0}\right]=x \cdot\left(D_{1}-3\left[P_{0}\right]\right)
$$

provided that a solution exists. Here $\operatorname{deg}\left(D_{1}\right)=\operatorname{deg}\left(D_{2}\right)=3$ and both $D_{1}$ and $D_{2}$ are sums of three $\mathbb{F}_{q}$-rational points: $D_{1}=\left[P_{1}^{1}\right]+\left[P_{2}^{1}\right]+\left[P_{3}^{1}\right], D_{2}=\left[P_{1}^{2}\right]+\left[P_{2}^{2}\right]+\left[P_{3}^{2}\right]$. For simplicity we assume that both divisors $D_{2}-3\left[P_{0}\right]$ and $D_{1}-3\left[P_{0}\right]$ live in the same subgroup of prime order $p$. Remark that, in general, any $\mathbb{F}_{q}$-rational divisor can be written in a unique way in the along $P_{0}$ maximally reduced form $D-\ell\left[P_{0}\right]$ ( $[\mathrm{Hes})$, where $\ell \leq 3$ and in the generic case $\ell=3$. If $D$ decomposes into a sum of three $\mathbb{F}_{q}$-rational points, it is called completely split. If the divisor is not completely split, the standard strategy is to multiply both sides of the DLP by constants until they become completely split and then proceed as usual. Once the discrete logarithm has been found, these multipliers must be cancelled. See [Die2] for details. Here is the algorithm as 
given in Die2 with one modification. Diem suggests taking the factor base to be slightly larger to ensure that the algorithm terminates successfully. The size used below is an absolute minimum for which we can expect the algorithm to succeed. In practice the size should be slightly bigger. Choosing the factor base: Choose a set $\mathcal{F} \subseteq \mathcal{C}\left(\mathbb{F}_{q}\right)$ with $\left\lceil((3 / 2) \ln q+4)^{1 / 2} q^{1 / 2}\right\rceil$ elements. Include the points $\left\{P_{j}^{i}\right\} \cup\left\{P_{0}\right\}$ in $\mathcal{F}$. The set $\mathcal{F}$ is called the factor base. Let $\mathcal{L}:=\mathcal{C}\left(\mathbb{F}_{q}\right) \backslash \mathcal{F}$ be the set of large primes.

Tree building: We constuct a tree $\mathbf{T}$ as follows.

Let $\mathbf{V}=\{*\}$ be the set of vertices of $\mathbf{T}$. Here $*$ denotes a distinguished special vertex. Let $\mathbf{E}=\emptyset$ be the set of edges.

for all unordered pairs $\left(F_{1}, F_{2}\right) \in \mathcal{F} \times \mathcal{F}$ such that $F_{1} \neq F_{2}$ do

Draw a line through the points $F_{1}$ and $F_{2}$. This will intersect the curve $\mathcal{C}$ at two other points $L_{1}$ and $L_{2}$.

if $L_{1}$ and $L_{2}$ are not both $\mathbb{F}_{q}$-rational then

Move on to the next pair.

end if

if $L_{1} \in \mathbf{V} \cup \mathcal{F}$ and $L_{2} \notin \mathbf{V} \cup \mathcal{F}$ then

Add a vertex to $\mathbf{T}$ labeled $L_{2}$.

If $L_{1} \in \mathbf{V}$ draw an edge to $\mathbf{T}$ connecting $L_{1}$ and $L_{2}$ and label the edge with the linear relation $\left[F_{1}\right]+\left[F_{2}\right]+\left[L_{1}\right]+\left[L_{2}\right]=0$. If $L_{1} \in \mathcal{F}$ draw an edge to $\mathbf{T}$ connecting $*$ and $L_{2}$ and label the edge with the linear relation $\left[F_{1}\right]+\left[F_{2}\right]+\left[L_{1}\right]+\left[L_{2}\right]=0$.

end if

if $\# \mathbf{V} \geq\left\lceil q^{3 / 4}\right\rceil$ then

break (tree building succeeded)

end if

end for

if $\# \mathbf{V}<\left\lceil q^{3 / 4}\right\rceil$ then

Tree building failed. Restart the algorithm with a different set $\mathcal{F}$. end if

Relation search:

for all unordered pairs $\left(F_{1}, F_{2}\right) \in \mathcal{F} \times \mathcal{F}, F_{1} \neq F_{2}$, that were not already used in tree building do

Draw a line through the points $F_{1}$ and $F_{2}$. This will intersect the curve $\mathcal{C}$ at two other points $L_{1}$ and $L_{2}$.

if $L_{1}$ and $L_{2}$ are not both $\mathbb{F}_{q}$-rational then

Move on to the next pair.

end if

if $L_{1}, L_{2} \in \mathbf{V} \cup \mathcal{F}$ then

If one or both of $L_{1}, L_{2}$ is in $\mathbf{V}$, use the tree and the linear relations labeling the edges to write $\left[L_{1}\right]$ and $\left[L_{2}\right]$ in terms of the factor base. A relation

$$
\sum_{F \in \mathcal{F}} \lambda_{F}[F]=0
$$

obtained in this way is called a full relation.

end if

if the number of full relations found is $\geq \# \mathcal{F}+1$ then

break (relation search succeeded)

end if

end for

if the number of full relations is $\leq \# \mathcal{F}$ then

Restart the algorithm with a different set $\mathcal{F}$. end if

\section{Linear algebra step:}

Construct a sparse matrix $M$ with $\# \mathcal{F}$ columns, each column labeled by a point of $\mathcal{F}$.

The first row of $M$ is given by the points in the divisor $D_{1}-3\left[P_{0}\right]$.

The second row of $M$ is given by the points in the divisor $D_{2}-3\left[P_{0}\right]$.

for each full relation do

Add a row to $M$ corresponding to the left-hand side of the full relation. 


\section{end for}

Use sparse linear algebra techniques to find a non-zero vector $\bar{v}$ such that $M \bar{v}=\overline{0}$ over the ring $\mathbb{Z} / p \mathbb{Z}$ where $p$ is the order of the subgroup of the Jacobian where the DLP was defined. Choose $\bar{v}$ to be such that the second component $v_{2}$ is invertible modulo $p$.

Output: $-v_{1} / v_{2}$ modulo $p$.

Theorem 1 ([Die2]). Under some heuristic assumptions and when $q$ is large enough, after a constant number of attempts the algorithm terminates successfully and outputs a number $x \in$ $\mathbb{Z} / p \mathbb{Z}$ such that $D_{2}-3\left[P_{0}\right]=x \cdot\left(D_{1}-3\left[P_{0}\right]\right)$. The complexity of the algorithm is $\widetilde{O}(q)$.

\section{Our Variant}

In our version of the algorithm we generate the factor base in a different way. We start with a much smaller initial set for the factor base of size $O\left(q^{1 / 8}\right)$, and then build up the factor base and the graph simultaneously at the beginning. This improves the efficiency of the graph-building, and then after this initial step we build the graph and find full relations simultaneously. As a result, both the overall relation collection time and the linear algebra stage are improved. Our algorithm works as follows.

Input:

1) The Jacobian of a smooth plane quartic $\mathcal{C}$ over a finite field $\mathbb{F}_{q}$;

2) An $\mathbb{F}_{q}$-rational point $P_{0}$ on the curve;

3) A discrete logarithm problem on the Jacobian

$$
D_{2}-3\left[P_{0}\right]=x \cdot\left(D_{1}-3\left[P_{0}\right]\right)
$$

where $\operatorname{deg}\left(D_{1}\right)=\operatorname{deg}\left(D_{2}\right)=3$ and both $D_{1}$ and $D_{2}$ are sums of three $\mathbb{F}_{q}$-rational points:

$$
D_{1}=\left[P_{1}^{1}\right]+\left[P_{2}^{1}\right]+\left[P_{3}^{1}\right], \quad D_{2}=\left[P_{1}^{2}\right]+\left[P_{2}^{2}\right]+\left[P_{3}^{2}\right] ;
$$

4) The size $p$ of a prime order subgroun ${ }^{3}$ containing $D_{2}-3\left[P_{0}\right]$ and $D_{1}-3\left[P_{0}\right]$.

Initialization: Let $\lambda$ be a positive real number satisfying

$$
\lambda \exp \left(4 \lambda^{8}\right)=q^{1 / 8} .
$$

Choose a set $\mathcal{R} \mathcal{P}$ of $\left\lceil 4 \lambda q^{1 / 8}\right\rceil \mathbb{F}_{q}$-rational points on the curve. Let $\mathcal{F}$ be another set of points, the factor base, and for now let

$$
\mathcal{F}:=\mathcal{R} \mathcal{P} \cup\left\{P_{j}^{i}\right\} \cup\left\{P_{0}\right\}
$$

Construction of the base vertices: We construct a graph $\mathbf{G}$ as follows.

Let $\mathbf{V}:=\{*\}$ be the set of vertices of the graph $\mathbf{G}$. Here $*$ denotes a distinguished special vertex. Let $\mathbf{E}:=\emptyset$ be the set of edges.

for all unordered pairs $\left(F_{1}, F_{2}\right) \in \mathcal{R} \mathcal{P} \times \mathcal{R} \mathcal{P}$ such that $F_{1} \neq F_{2}$ do

Draw a line through the points $F_{1}$ and $F_{2}$. This will intersect the curve $\mathcal{C}$ at two other points $F$ and $L$.

if $F$ and $L$ are $\mathbb{F}_{q}$-rational, $L \notin \mathbf{V} \cup \mathcal{F}$ and $F \notin \mathbf{V}$ then

Let $\mathcal{F}:=\{F\} \cup \mathcal{F}$ and add a vertex to $\mathbf{V}$ labeled $L$.

Add an edge to $\mathbf{E}$ connecting $*$ and $L$ and label the edge with the linear relation

$\left[F_{1}\right]+\left[F_{2}\right]+[F]+[L]=0$. The vertices $L$ constructed here we call base vertices. end if

end for

Let $\mathbf{B}$ denote the set of all base vertices. Note that at this point $\mathbf{V}=\mathbf{B} \cup\{*\}$.

\section{Construction of the triangle relations:}

for all unordered pairs $\left(B_{1}, B_{2}\right) \in \mathbf{B} \times \mathbf{B}$ such that $B_{1} \neq B_{2}$ do

Draw a line through the points $B_{1}$ and $B_{2}$. This will intersect the curve $\mathcal{C}$ at two other points $F$ and $L$.

if $F$ and $L$ are $\mathbb{F}_{q}$-rational, $L \notin \mathbf{V} \cup \mathcal{F}$ and $F \notin \mathbf{V}$ then

\footnotetext{
${ }^{3}$ It is not strictly speaking necessary for the subgroup to have prime order, but this will simplify the linear algebra step and guarantee that the DLP has a solution.
} 
Let $\mathcal{F}:=\{F\} \cup \mathcal{F}$ and add a vertex to $\mathbf{V}$ labeled $L$.

Draw a triangle in the graph with corners $B_{1}, B_{2}$ and $L$. Label the triangle with the

linear relation $\left[B_{1}\right]+\left[B_{2}\right]+[L]+[F]=0$. The vertices $L$ constructed here we call top

triangle vertices.

end if

end for

Graph building and relation search (RS):

for all unordered pairs $\left(F_{1}, F_{2}\right) \in \mathcal{F} \times \mathcal{F}$ such that $F_{1} \neq F_{2}$ do

Draw a line through the points $F_{1}$ and $F_{2}$. This will intersect the curve $\mathcal{C}$ at two other points $L_{1}$ and $L_{2}$.

if $L_{1}$ and $L_{2}$ are not both $\mathbb{F}_{q}$-rational then

Move on to the next pair.

end if

if $L_{1} \in \mathbf{V} \backslash \mathbf{B}$ and $L_{2} \notin \mathbf{V} \cup \mathcal{F}$ then

Add a vertex to $\mathbf{V}$ labeled $L_{2}$.

Add an edge to $\mathbf{E}$ connecting $L_{1}$ and $L_{2}$ and label the edge with the linear relation

$\left[F_{1}\right]+\left[F_{2}\right]+\left[L_{1}\right]+\left[L_{2}\right]=0$.

else if $L_{1}, L_{2} \in(\mathbf{V} \backslash \mathbf{B}) \cup \mathcal{F}$ then

In case $L_{1}, L_{2} \in \mathbf{V} \backslash \mathbf{B}$, use the graph and the linear relations labeling the edges to write $\left[L_{1}\right]$ and $\left[L_{2}\right]$ in terms in the factor base to obtain a relation

$$
\lambda_{1}\left[L_{1}^{\prime}\right]+\lambda_{2}\left[L_{2}^{\prime}\right]+\sum_{F \in \mathcal{F}} \lambda_{F}[F]=0
$$

where $L_{1}^{\prime}$ and $L_{2}^{\prime}$ are top triangle vertices, $\lambda_{i}$ are \pm 1 and $\lambda_{F}$ are integers. Then use the triangle relations to substitute $L_{1}^{\prime}$ and $L_{2}^{\prime}$ with elements of $\mathcal{F}$. In cases where neither one or exactly one of $L_{i}$ is in $\mathbf{V} \backslash \mathbf{B}$ we need to perform the above substitution process only to the one that is in $\mathbf{V} \backslash \mathbf{B}$. In any case we obtain a relation involving only elements of $\mathcal{F}$, which we record. We call it a full relation.

end if

if the number of full relations found is $\geq \# \mathcal{F}+1$ then

break (relation search succeeded)

end if

end for

if the number of full relations is $\leq \# \mathcal{F}$ then

Restart the algorithm.

end if

In practice we do not restart the algorithm but instead re-run the for-loop. Almost certainly it will break very soon after starting and only a few if any duplicate full relations are produced.

\section{Linear algebra step:}

Construct a sparse matrix $M$ with $\# \mathcal{F}$ columns, each column labeled by a point of $\mathcal{F}$.

The first row of $M$ is given by the points in the divisor $D_{1}-3\left[P_{0}\right]$.

The second row of $M$ is given by the points in the divisor $D_{2}-3\left[P_{0}\right]$.

for each full relation do

Add a row to $M$ corresponding to the left-hand side of the full relation end for

Use sparse linear algebra techniques to find a non-zero vector $\bar{v}$ such that $M \bar{v}=\overline{0}$ over the ring $\mathbb{Z} / p \mathbb{Z}$ where $p$ is the order of the subgroup of the Jacobian where the DLP was defined. Choose $\bar{v}$ to be such that the second component $v_{2}$ is invertible modulo $p$.

Output: $-v_{1} / v_{2}$ modulo $p$.

Theorem 2. (Heuristic) Under some heuristic assumptions and if $q$ is large enough, after a constant number of attempts the algorithm terminates and outputs a number $x \in \mathbb{Z} / p \mathbb{Z}$ such that $D_{2}-3\left[P_{0}\right]=x \cdot\left(D_{1}-3\left[P_{0}\right]\right)$. The size of the factor base will be approximately $4 \lambda^{4} q^{1 / 2}$ and the size of the graph will be approximately $4 \lambda^{2} q^{3 / 4}$.

Proof. Correctness is easy; see for example [Die2]. It remains to prove that the size of the factor base is sufficiently large for the algorithm to terminate. The strategy is the following. Let $N$ be 
the size of the factor base at the beginning, essentially $N=\# \mathcal{R} \mathcal{P}$. First we compute the number of factor base elements and graph vertices produced when constructing the base vertices and the triangle relations, and express these in terms of $N$. Next we compute the expected number of full relations produced in the graph building step when allowing the graph to grow until it has $N_{\text {max }}$ vertices. Since we know that the number of full relations needed is $\# \mathcal{F}+1$, we can find an expression for $N_{\max }$ in terms of $N$. Finally we compute the number of factor base pairs needed to grow the graph to size $N_{\max }$. We set this number equal to the number of factor base pairs available, which yields an equation for $N$.

Due to the roundabout way of computing the numbers $N$ and $N_{\max }$, we need to have an idea of their sizes beforehand, so that the most significant terms can be computed. For this purpose, we assume $N=O\left(q^{1 / 8}\right)$, so $\# \mathcal{F}=O\left(q^{1 / 2}\right)$, and $N_{\max }=O\left(q^{3 / 4}\right)$, which are in line with Diem's choices in Die2.

By [DT, Prop. 14], a line through two $\mathbb{F}_{q}$-rational points on the curve intersects the curve at two other $\mathbb{F}_{q}$-rational points with probability $1 / 2+O\left(q^{-1 / 2}\right)$.

Suppose $\mathcal{R P}$ is a set of $N=O\left(q^{1 / 8}\right)$ random $\mathbb{F}_{q}$-rational points on the curve. Construction of the base vertices $\mathbf{B}$ produces

$$
\left(\frac{1}{2}+O\left(q^{-1 / 2}\right)\right)\left(\begin{array}{c}
N \\
2
\end{array}\right)=\frac{N^{2}}{4}+O\left(q^{1 / 8}\right)
$$

base vertices and equally many factor base elements.

Construction of the triangle relations from the set $\mathbf{B}$ produces

$$
\left(\frac{1}{2}+O\left(q^{-1 / 2}\right)\right)\left(\begin{array}{c}
\# \mathbf{B} \\
2
\end{array}\right)=4\left(\frac{N}{4}\right)^{4}+O\left(q^{3 / 8}\right)
$$

triangles and equally many factor base elements. At this point we expect to have

$$
\# \mathcal{F}=4\left(\frac{N}{4}\right)^{4}+O\left(q^{3 / 8}\right)=4\left(\frac{N}{4}\right)^{4}\left(1+O\left(q^{-1 / 8}\right)\right) \text {. }
$$

We will need to choose $N$ so that in the graph building step we expect to find $\# \mathcal{F}+1$ full relations. To this end, suppose that when the algorithm terminates successfully the number of vertices in the graph is $N_{\max }=O\left(q^{3 / 4}\right)$. When the graph building starts we already have approximately $4(N / 4)^{4}$ vertices in the graph, consisting of the base vertices $\mathbf{B}$ and the top triangle vertices. If the size of the graph at a particular moment is $x$, the probability of adding a new vertex and edge to the graph with a particular choice of a pair $\left(F_{1}, F_{2}\right) \in \mathcal{F} \times \mathcal{F}$ is

$$
\left(1+O\left(q^{-1 / 2}\right)\right) \frac{x}{q}\left(1-\frac{x}{q}\right)
$$

Hence, to add one new vertex and edge we need to try approximately

$$
\left(1+O\left(q^{-1 / 2}\right)\right) \frac{1}{(x / q)(1-x / q)}
$$

pairs. For each pair we try, the probability of finding a full relation through the process described in the algorithm is

$$
\left(\frac{1}{2}+O\left(q^{-1 / 2}\right)\right)\left(\frac{x}{q}\right)^{2}
$$

so when the size of the graph is increased by one we expect to have found approximately

$$
\left(\frac{1}{2}+O\left(q^{-1 / 2}\right)\right) \frac{x / q}{1-x / q}
$$

more full relations.

Once the triangle building step has been completed, the size of the graph is roughly equal to the number of triangles, which again is roughly equal to the size of the factor base. We denote this by

$$
N_{0}=4\left(\frac{N}{4}\right)^{4}+O\left(q^{3 / 8}\right)
$$

Note that once the triangles have been constructed, the factor base does not change anymore and only the graph is built using the factor base. The total number of full relations produced in the entire graph building step, when the size of the graph is built from $N_{0}$ to $N_{\max }=O\left(q^{3 / 4}\right)$, is

$$
\left(\frac{1}{2}+O\left(q^{-1 / 2}\right)\right) \sum_{k=N_{0}}^{N_{\max }-1} \frac{k / q}{1-k / q}=\left(\frac{1}{2}+O\left(q^{-1 / 2}\right)\right)\left(\int_{N_{0}}^{N_{\max }} \frac{x / q}{1-x / q} d x+E\right)
$$




$$
=\left(\frac{q}{2}+O\left(q^{1 / 2}\right)\right)\left[-\frac{N_{\max }}{q}-\ln \left(1-\frac{N_{\max }}{q}\right)+\frac{N_{0}}{q}+\ln \left(1-\frac{N_{0}}{q}\right)+\frac{E}{q}\right],
$$

where the error term $E$ is

$$
E=\sum_{k=N_{0}}^{N_{\max }-1}\left(\frac{k / q}{1-k / q}-\int_{k}^{k+1} \frac{x / q}{1-x / q} d x\right)
$$

Since the function $(k / q) /(1-k / q)$ is increasing, $E$ satisfies

$$
\sum_{k=N_{0}}^{N_{\max }-1}\left(\frac{k / q}{1-k / q}-\frac{(k+1) / q}{1-(k+1) / q}\right)=\frac{N_{0} / q}{1-N_{0} / q}-\frac{N_{\max } / q}{1-N_{\max } / q} \leq E \leq 0 .
$$

Hence $E \in O\left(q^{-1 / 4}\right)$ and $E / q \in O\left(q^{-5 / 4}\right)$. If we expand out the first two terms of the logarithms in (1), we find that the total number of full relations produced in the entire graph building step is

$$
\left(\frac{q}{2}+O\left(q^{1 / 2}\right)\right)\left[\frac{1}{2}\left(\frac{N_{\max }}{q}\right)^{2}+O\left(q^{-3 / 4}\right)\right]=\frac{q}{4}\left(\frac{N_{\max }}{q}\right)^{2}+O\left(q^{1 / 4}\right) .
$$

We want this to equal roughly the size of the factor base (or maybe slightly more in practice to ensure that the algorithm terminates successfully) so we must have

$$
\frac{q}{4}\left(\frac{N_{\max }}{q}\right)^{2}=4\left(\frac{N}{4}\right)^{4}\left(1+O\left(q^{-1 / 8}\right)\right)
$$

From this we can solve

$$
\frac{N_{\max }}{4 q}=\left(\frac{N}{4 q^{1 / 4}}\right)^{2}\left(1+O\left(q^{-1 / 8}\right)\right)=\left(\frac{N}{4 q^{1 / 4}}\right)^{2}+O\left(q^{-3 / 8}\right) .
$$

With this we are ready to compute the value of $N$. The number of unordered pairs of factor base elements needed to build the graph from size $N_{0}$ to size $N_{\max }=O\left(q^{3 / 4}\right)$ should equal the number of pairs available, i.e. $\left(\begin{array}{c}\# \mathcal{F} \\ 2\end{array}\right)=8(N / 4)^{8}+O\left(q^{7 / 8}\right)$. Therefore,

$$
\begin{gathered}
8\left(\frac{N}{4}\right)^{8}+O\left(q^{7 / 8}\right)=\left(1+O\left(q^{-1 / 2}\right)\right) \sum_{k=N_{0}}^{N_{\max }-1} \frac{1}{(k / q)(1-k / q)} \\
=\left(1+O\left(q^{-1 / 2}\right)\right)\left(\int_{N_{0}}^{N_{\max }} \frac{1}{(x / q)(1-x / q)} d x+E\right) .
\end{gathered}
$$

The error term $E$ is

$$
E=\sum_{k=N_{0}}^{N_{\max }-1}\left(\frac{1}{(k / q)(1-k / q)}-\int_{k}^{k+1} \frac{1}{(x / q)(1-x / q)} d x\right) .
$$

Since the function $1 /((k / q)(1-k / q))$ is decreasing, $E$ satisfies

$$
0 \leq E \leq \frac{1}{\left(N_{0} / q\right)\left(1-N_{0} / q\right)}-\frac{1}{\left(N_{\max } / q\right)\left(1-N_{\max } / q\right)}
$$

so $E \in O\left(q^{1 / 2}\right)$. Now (3) becomes

$$
\begin{gathered}
\left(q+O\left(q^{1 / 2}\right)\right)\left[\ln \left(\frac{N_{\max }}{4 q}\right)-\ln \left(1-\frac{N_{\max }}{q}\right)-\ln \left(\frac{N_{0}}{4 q}\right)+\ln \left(1-\frac{N_{0}}{q}\right)+\frac{E}{q}\right] \\
=\left(q+O\left(q^{1 / 2}\right)\right)\left[\ln \left(\frac{N_{\max }}{4 q}\right)-\ln \left(\frac{N_{0}}{4 q}\right)+O\left(q^{-1 / 4}\right)\right] \\
=-2 q \ln \left(\frac{N}{4 q^{1 / 4}}\right)+O\left(q^{7 / 8}\right)
\end{gathered}
$$

where we have used (2). Hence we obtain the equation

$$
4\left(\frac{N}{4 q^{1 / 8}}\right)^{8}+O\left(q^{-1 / 8}\right)=-\ln \left(\frac{N}{4 q^{1 / 4}}\right) .
$$

Denoting

$$
\lambda=\frac{N}{4 q^{1 / 8}}
$$


this becomes

$$
\lambda \exp \left(4 \lambda^{8}\right)=q^{1 / 8}+O(1) \approx q^{1 / 8},
$$

where the approximation makes sense when $q$ is large enough. In practice the error term is small, even for small field sizes. The function $\lambda \exp \left(4 \lambda^{8}\right)-q^{1 / 8}$ is monotonically increasing and has precisely one positive real root. This is the equation stated in the initialization step of the algorithm, so if we take $N=4 \lambda q^{1 / 8}$ the algorithm can be expected to terminate successfully. If $q$ is large, then the size of the factor base will be

$$
\# \mathcal{F}=4 \lambda^{4} q^{1 / 2}+O\left(q^{3 / 8}\right) \approx 4 \lambda^{4} q^{1 / 2}
$$

and the size of the graph when the algorithm terminates will be

$$
N_{\max }=4 \lambda^{2} q^{3 / 4}+O\left(q^{5 / 8}\right) \approx 4 \lambda^{2} q^{3 / 4}
$$

For field sizes of most practical interest (perhaps between 60 and 120 bits) our algorithm has not much worse storage requirements than Diem's algorithm but our factor base remains smaller. In practice the factor base can be taken to be even slightly smaller than $4 \lambda^{4} q^{1 / 2}$ if we run the graph building step twice in a row as was explained earlier. We will look at some precise numbers in the next section.

\section{Specifics of Implementation in Characteristic 2}

In our experiments we made an artificial restriction to the case of binary fields in order to be able to count the number of points on the Jacobian easily. In this case it is easy to perform the geometric step of the algorithm where a line is drawn through two points on the curve and the intersection divisor is computed. Indeed, we do this by first constructing an equation for the line, then solving for one of the variables in terms of the other ones and substituting this into the equation of the curve to obtain a quartic polynomial in one variable. It is a simple computation to divide this polynomial by the two linear factors corresponding to the two points we started with. Finally the quadratic equation can be transformed into an Artin-Schreier equation by a linear change of variables and the solutions can be immediately written down using Chen's formulas. The complexity is logarithmic in $q$.

For odd characteristic fields one has to compute a square-root in $\mathbb{F}_{q}$ using the Tonelli-Shanks algorithm to solve the quadratic polynomial, the complexity of which is logarithmic in $q$.

\section{Complexity for Realistic Field Sizes}

\section{Relation Collection Time and Total Memory}

In our naive implementation the number of field multiplications (in the binary field case) needed to process each pair of factor base elements as explained above was

$$
M_{\text {pair }}:=7 \log _{2} q+13
$$

This count is an actual count of how many field multiplications are used to process each pair of points in our code, but it is also a theoretical count of the number of field operations required to pass a line through two points and intersect it with the curve. There are field inversions involved, which roughly explains the $\log q$ factors. This number could likely be improved in a more robust implementation. The total number of field multiplications needed was therefore approximately

$$
M_{\text {Total }} \approx M_{\text {pair }} \cdot\left(\begin{array}{c}
\# \mathcal{F} \\
2
\end{array}\right)=\left(7 \log _{2} q+13\right) \cdot 8 \lambda^{8} q .
$$

Remark 1. Locally the numbers $\lambda$ behave roughly logarithmically as a function of $q$. If we focus on the range $q \in\left[2^{70}, 2^{120}\right]$ we can for instance approximate

$$
\lambda(q) \approx C \log _{2}^{\alpha} q, \quad \text { where } \quad C=0.62054, \quad \alpha=0.12431 .
$$

Then

$$
M_{\text {Total }} \approx 0.17589 \cdot\left(7 \log _{2} q+13\right) \cdot \log _{2}^{0.99448}(q) \cdot q \approx 1.23123 \cdot \log _{2}^{2}(q) \cdot q
$$


Table 1: Results for field sizes of practical interest

\begin{tabular}{c|c|c|c|c|c|c} 
Field size & $\lambda$ & $\log _{q} \# \mathcal{F}$ & $\log _{q} N_{\max }$ & $\log _{q} M_{\text {Total }}$ & $\log _{2} M_{\text {Total }}$ & Memory \\
\hline $2^{30}$ & 0.94987 & 0.55677 & 0.81172 & 1.34024 & 40.20729 & $7.4 \mathrm{~GB}$ \\
$2^{40}$ & 0.98285 & 0.54750 & 0.79875 & 1.27488 & 50.99510 & $1430 \mathrm{~GB}$ \\
$2^{50}$ & 1.00974 & 0.54112 & 0.79056 & 1.23231 & 61.61568 & $267 \mathrm{~TB}$ \\
$2^{60}$ & 1.03251 & 0.53641 & 0.78487 & 1.20212 & 72.12744 & $50490 \mathrm{~TB}$ \\
$2^{70}$ & 1.05230 & 0.53277 & 0.78067 & 1.17947 & 82.56275 & $1.90 \cdot 10^{7} \mathrm{~TB}$ \\
$2^{80}$ & 1.06983 & 0.52987 & 0.77743 & 1.16177 & 92.94144 & $3.56 \cdot 10^{9} \mathrm{~TB}$ \\
$2^{90}$ & 1.08559 & 0.52749 & 0.77486 & 1.14752 & 103.27649 & $6.62 \cdot 10^{11} \mathrm{~TB}$ \\
$2^{100}$ & 1.09992 & 0.52550 & 0.77275 & 1.13577 & 113.57690 & $1.2 \cdot 10^{14} \mathrm{~TB}$ \\
$2^{110}$ & 1.11306 & 0.52380 & 0.77099 & 1.12590 & 123.84916 & $2.28 \cdot 10^{16} \mathrm{~TB}$ \\
$2^{115}$ & 1.11926 & 0.52304 & 0.77022 & 1.12153 & 128.97628 & $3.10 \cdot 10^{17} \mathrm{~TB}$ \\
$2^{120}$ & 1.12522 & 0.52234 & 0.76950 & 1.11748 & 134.09806 & $4.22 \cdot 10^{18} \mathrm{~TB}$ \\
$2^{140}$ & 1.14712 & 0.51994 & 0.76711 & 1.10386 & 154.53975 & $2.16 \cdot 10^{23} \mathrm{~TB}$ \\
$2^{160}$ & 1.16646 & 0.51805 & 0.76528 & 1.09327 & 174.92298 & $7.29 \cdot 10^{27} \mathrm{~TB}$ \\
$2^{180}$ & 1.18380 & 0.51652 & 0.76382 & 1.08479 & 195.26141 & $8.43 \cdot 10^{31} \mathrm{~TB}$ \\
$2^{200}$ & 1.19954 & 0.51525 & 0.76262 & 1.07782 & 215.56441 & $1.10 \cdot 10^{37} \mathrm{~TB}$ \\
$2^{220}$ & 1.21397 & 0.51418 & 0.76163 & 1.07199 & 235.83869 & $3.71 \cdot 10^{41} \mathrm{~TB}$ \\
$2^{240}$ & 1.22729 & 0.51326 & 0.76080 & 1.06704 & 256.08921 & $1.24 \cdot 10^{46} \mathrm{~TB}$ \\
\hline
\end{tabular}

The memory consumption was roughly 370 bytes per graph vertex but our implementation was not optimized towards saving memory so this number can probably be reduced by a significant factor. Moreover, every vertex data must contain the coordinates of some points on the curve or other vertex identifiers, the size of which grows logarithmically in $q$. Hence the size of a vertex will grow logarithmically in $q$, but this dependence is weak and for practical field sizes it is not a significant factor. To take this into account, we assume the size of a vertex data is $\left\lceil\left(\log _{2} q\right) / 64\right\rceil \cdot 370$ bytes. The results are shown in Table 1

According to these estimates one should use a field of size at least 115 bits to get a security level of 128 bits and a field of size at least 240 bits to get a security level of 256 bits. Of course this is only the relation collection step and is not taking into account the linear algebra step or even more importantly the massive memory consuption. Later we will see that there is a timememory trade-off which can be used to reduce the memory cost significantly without affecting the computational complexity much, and that parallelization can be used to even further reduce both time and memory requirements (per computer).

\section{Experimental Results for Small Examples}

We ran small experiments and got results corresponding to the theoretical results above. We chose $\mathcal{R} \mathcal{P}$ to be slightly bigger than was strictly speaking needed to ensure that the algorithm finishes successfully on the first attempt. More precisely, we chose it so that about $95 \%$ of the factor base pairs are used when the algorithm finishes. The results are shown in Table 2 We conclude that the experimental results correspond closely to the theoretical results, even for such small field sizes.

\section{Linear Algebra}

We recall that the complexity of sparse linear algebra algorithms is $O\left(w \cdot(\# \mathcal{F})^{2}\right)$ where $w$ denotes the average row weight. The row weight depends logarithmically on the field size. The expected average depth of a tree on a given number of vertices can be estimated using the method in GTTD but we need to take into account that the size of the graph is changing while we are looking for full relations.

Lemma 1. The average row weight of the matrix is $w \leq 18-8 \ln \lambda+\ln q$.

Proof. In GTTD it is established that for a tree containing $k$ vertices the expected average depth can be approximated from above by the function $1+\ln k$. Our graph consists of trees built on top of each of the top triangle vertices. When our graph has a total of $k$ vertices, each one of these $4 \lambda^{4} q^{1 / 2}+O\left(q^{3 / 8}\right)$ trees has on average $k /\left(4 \lambda^{4} q^{1 / 2}\right)+O\left(q^{-1 / 8}\right)$ vertices. So if we choose a 
Table 2: Experimental results. (FBPU := percent of factor base pairs used)

Field size $\log _{q} \# \mathcal{F}$ (theory) $\mid \log _{q} \# \mathcal{F}$ (practice) $\mid$ FBPU $\log _{q} M_{\text {Total }}$ (theory) $\mid \log _{q} M_{\text {Total }}$ (practice)

\begin{tabular}{l|l|l|l|l|l}
$2^{17}$ & 0.57846 & 0.58034 & 96.4 & 1.51247 & 1.50780 \\
$2^{19}$ & 0.57387 & 0.57692 & 95.5 & 1.47352 & 1.46740 \\
$2^{21}$ & 0.56683 & 0.57223 & 95.3 & 1.44080 & 1.43510 \\
$2^{23}$ & 0.56637 & 0.56819 & 95.4 & 1.41287 & 1.40769 \\
$2^{25}$ & 0.56325 & 0.56468 & 96.0 & 1.38869 & 1.38418 \\
$2^{27}$ & 0.56046 & 0.56158 & 96.4 & 1.36752 & 1.36351 \\
\hline
\end{tabular}

tree at random and a point in it at random, the expected depth (measured from the root of that tree) is

$$
\leq 1+\ln \left(\frac{k}{4 \lambda^{4} q^{1 / 2}}\right)+O\left(\frac{q^{3 / 8}}{k}\right)=1+\ln \left(\frac{k}{4 \lambda^{4} q^{1 / 2}}\right)+O\left(q^{-3 / 8}\right) .
$$

We find full relations while building the graph so the sizes of the trees change. Recall that right after the triangles are constructed, the size of the graph is

$$
N_{0}=4 \lambda^{4} q^{1 / 2}+O\left(q^{3 / 8}\right) .
$$

When a full relation is produced and the graph tracing step performed, the number of factor base elements we end up with on average i: 4

$$
\begin{aligned}
& \leq \frac{2^{2}}{\# \mathcal{F}} \sum_{k=N_{0}}^{N_{\max }-1}\left(1+\ln \left(\frac{k}{4 \lambda^{4} q^{1 / 2}}\right)+O\left(q^{-3 / 8}\right)\right) \cdot\left(\frac{1}{2}+O\left(q^{-1 / 2}\right)\right) \frac{k / q}{1-k / q} \\
& =\frac{q^{1 / 2}}{2 \lambda^{4}}\left(1+O\left(q^{-1 / 8}\right)\right)\left[\int_{N_{0} / q}^{N_{\max } / q}\left(1+\ln \left(\frac{t q^{1 / 2}}{4 \lambda^{4}}\right)+O\left(q^{-3 / 8}\right)\right) \cdot \frac{t}{1-t} d t+\frac{E}{q}\right] \\
& =\frac{q^{1 / 2}}{2 \lambda^{4}}\left(1+O\left(q^{-1 / 8}\right)\right)\left[\left(1+O\left(q^{-1 / 4}\right)\right) \int_{N_{0} / q}^{N_{\max } / q} t\left(1+\ln \left(\frac{t q^{1 / 2}}{4 \lambda^{4}}\right)+O\left(q^{-3 / 8}\right)\right) d t+\frac{E}{q}\right] \\
& =4\left(1+O\left(q^{-1 / 8}\right)\right)\left[\left(\frac{1}{2}-2 \ln \lambda+\frac{1}{4} \ln q+O\left(q^{-1 / 8}\right)\right)+O\left(q^{-1 / 2}\right)+\frac{E}{8 \lambda^{4} q^{1 / 2}}\right] .
\end{aligned}
$$

Here the error term $E$ is

$$
\begin{gathered}
E=\sum_{k=N_{0}}^{N_{\max }-1}\left[\left(1+\ln \left(\frac{k}{4 \lambda^{4} q^{1 / 2}}\right)+O\left(q^{-3 / 8}\right)\right) \frac{k / q}{1-k / q}\right. \\
\left.-\int_{k}^{k+1}\left(1+\ln \left(\frac{x}{4 \lambda^{4} q^{1 / 2}}\right)+O\left(q^{-3 / 8}\right)\right) \frac{x / q}{1-x / q} d x\right]
\end{gathered}
$$

and it satisfies the bounds

$$
\begin{gathered}
\left(1+\ln \left(\frac{N_{0}}{4 \lambda^{4} q^{1 / 2}}\right)+O\left(q^{-3 / 8}\right)\right) \frac{N_{0} / q}{1-N_{0} / q} \\
-\left(1+\ln \left(\frac{N_{\max }}{4 \lambda^{4} q^{1 / 2}}\right)+O\left(q^{-3 / 8}\right)\right) \frac{N_{\max } / q}{1-N_{\max } / q} \leq E \leq 0 .
\end{gathered}
$$

Hence $E \in O\left(q^{-1 / 4} \ln q\right)$ and $E /\left(8 \lambda^{4} q^{1 / 2}\right) \in O\left(q^{-3 / 4} \ln q\right)$. Finally with this we find that the average number of factor base elements appearing is

$$
2-8 \ln \lambda+\ln q+O\left(q^{-1 / 8} \ln q\right)+O\left(q^{-3 / 4} \ln q\right)=2-8 \ln \lambda+\ln q+O\left(q^{-1 / 8} \ln q\right) .
$$

In addition to the contribution coming from moving in the graph, we have 2 initial factor base elements, 2 coming from using the triangle relations and 12 from the base vertices. The expected average row weight should therefore satisfy $w \leq 18-8 \ln \lambda+\ln q$.

${ }^{4}$ One factor of 2 is for the two factor base elements that are produced at every step in the graph and the other factor of 2 from tracing back two vertices to their respective roots. Here we are only concerned with the factor base elements that result from moving in the graph. The others are taken into account below. 
Note that in practice $q$ is large so $q^{-1 / 8} \ln q$ is small, and we let $w_{\text {est }}:=18-8 \ln \lambda+\ln q$. Results for field sizes of practical interest are shown in Table 3 The complexity of the linear algebra is in all cases slightly better than the complexity of relation search so there might be some room for optimization by choosing the factor base to be slightly larger, which makes relation collection faster and linear algebra slower.

Table 3: Linear algebra results for field sizes of practical interest

\begin{tabular}{c|c|c|c|c} 
Field size & $\log _{2} w_{\text {est }}$ & $\log _{2} \# \mathcal{F}$ & Lin. alg. & $M_{\text {Total }}$ \\
\hline $2^{30}$ & 5.29300 & 16.70320 & $\approx 2^{39}$ & $\approx 2^{40}$ \\
$2^{40}$ & 5.51930 & 21.90017 & $\approx 2^{49}$ & $\approx 2^{51}$ \\
$2^{50}$ & 5.71644 & 27.05593 & $\approx 2^{60}$ & $\approx 2^{62}$ \\
$2^{60}$ & 5.89076 & 32.18461 & $\approx 2^{70}$ & $\approx 2^{72}$ \\
$2^{70}$ & 6.04685 & 37.29417 & $\approx 2^{81}$ & $\approx 2^{83}$ \\
$2^{80}$ & 6.18808 & 42.38952 & $\approx 2^{91}$ & $\approx 2^{93}$ \\
$2^{90}$ & 6.31698 & 47.47391 & $\approx 2^{101}$ & $\approx 2^{103}$ \\
$2^{100}$ & 6.43551 & 52.54957 & $\approx 2^{112}$ & $\approx 2^{114}$ \\
$2^{110}$ & 6.54518 & 57.61814 & $\approx 2^{122}$ & $\approx 2^{124}$ \\
$2^{115}$ & 6.59709 & 60.15016 & $\approx 2^{127}$ & $\approx 2^{129}$ \\
$2^{120}$ & 6.64723 & 62.68083 & $\approx 2^{132}$ & $\approx 2^{134}$ \\
$2^{140}$ & 6.83216 & 72.79205 & $\approx 2^{152}$ & $\approx 2^{155}$ \\
$2^{160}$ & 6.99630 & 82.88852 & $\approx 2^{173}$ & $\approx 2^{175}$ \\
$2^{180}$ & 7.14381 & 92.97370 & $\approx 2^{193}$ & $\approx 2^{195}$ \\
$2^{200}$ & 7.27774 & 103.04993 & $\approx 2^{213}$ & $\approx 2^{216}$ \\
$2^{220}$ & 7.40038 & 113.11892 & $\approx 2^{234}$ & $\approx 2^{236}$ \\
$2^{240}$ & 7.51347 & 123.18192 & $\approx 2^{254}$ & $\approx 2^{256}$ \\
\hline
\end{tabular}

\section{Time-Memory Trade-offs}

Our variant presented in Section 3 improves on the computational complexity of Diem's algorithm by building a larger graph. Since the memory costs of algorithms of this type are enormous, one should ask how the computational complexity behaves when the size of the graph is limited to something between that of Diem's algorithm ( $q^{3 / 4}$ vertices) and $N_{\text {max }}$ given in Theorem 2 .

Let $\chi$ be a fixed positive real number. Suppose in our algorithm we let our graph grow to size $\chi q^{3 / 4}$ and after that only search for full relations. More precisely, we start by choosing a set $\mathcal{R} \mathcal{P}_{\chi}$ of $4 \eta_{\chi} q^{1 / 8}$ random points on the curve where $\eta_{\chi}$ is a real number depending on $\chi$ and use these to construct a factor base $\mathcal{F}_{\chi}$ of size $4 \eta_{\chi}^{4} q^{1 / 2}$ just as in our original algorithm. We expect $\eta_{\chi}$ to be somewhere between 1 and 10 for practical field sizes. In the graph building/relation searching step we stop adding new vertices to the graph once it has reached size $\chi q^{3 / 4}$ and after that only search for full relations. To simplify the notation we denote $\eta_{\chi}$ just by $\eta$.

Theorem 3. (Heuristic) If $q$ is large enough and we take $\eta$ to be a root of

$$
2 \eta^{2} \exp \left(4 \eta^{8}-4 \eta^{4} / \chi^{2}+1 / 4\right)=\chi^{1 / 2} q^{1 / 8}
$$

we can expect the algorithm to terminate successfully. The average row weight of the matrix will be approximately

$$
w_{e s t}^{\chi}:=20-\frac{\chi^{2}}{8 \eta^{4}}-4 \ln \left(4 \eta^{4}\right)+\ln q+4 \ln \chi .
$$

Proof. This is very similar to the proof of Theorem 2 We start with a set $\mathcal{R} \mathcal{P}_{\chi}$ of $4 \eta q^{1 / 8}=$ $O\left(q^{1 / 8}\right)$ random points. The size of the factor base and the number of vertices in the graph after the triangles have been constructed are

$$
\# \mathcal{F}_{\chi}=4 \eta^{4} q^{1 / 2}+O\left(q^{3 / 8}\right), \quad N_{0}=4 \eta^{4} q^{1 / 2}+O\left(q^{3 / 8}\right)
$$


We build the graph until it has size $\chi q^{3 / 4}$. This produces

$$
\left(\frac{1}{2}+O\left(q^{-1 / 2}\right)\right) \sum_{k=N_{0}}^{\chi q^{3 / 4}-1} \frac{k / q}{1-k / q}=\frac{\chi^{2} q^{1 / 2}}{4}+O\left(q^{1 / 4}\right)
$$

full relations (see the proof of Theorem 2). The total number of full relations needed is $\# \mathcal{F}_{\chi}+1$ so we are lacking

$$
4 \eta^{4} q^{1 / 2}-\frac{\chi^{2} q^{1 / 2}}{4}+O\left(q^{3 / 8}\right)=\left(4 \eta^{4}-\frac{\chi^{2}}{4}\right) q^{1 / 2}+O\left(q^{3 / 8}\right)
$$

of them. We also need to know how many pairs of factor base elements were used in this. Just like in the proof of Theorem 2 we find that this number is

$$
\left(1+O\left(q^{-1 / 2}\right)\right) \sum_{k=N_{0}}^{\chi q^{3 / 4}-1} \frac{1}{(k / q)(1-k / q)}=q \ln \left(\frac{\chi q^{1 / 4}}{4 \eta^{4}}\right)+O\left(q^{7 / 8}\right) .
$$

Now once the graph has size $\chi q^{3 / 4}$ we need to find the rest of the full relations. For each pair of factor base elements we try, the probability of finding a full relation is now approximately $\chi^{2} /\left(2 q^{1 / 2}\right)+O\left(q^{-1}\right)$ (see the proof of Theorem 2). Thus we need to try

$$
\frac{2 q^{1 / 2}}{\chi^{2}}\left(1+O\left(q^{-1 / 2}\right)\right) \cdot\left[\left(4 \eta^{4}-\frac{\chi^{2}}{4}\right) q^{1 / 2}+O\left(q^{3 / 8}\right)\right]=\left(\frac{8 \eta^{4}}{\chi^{2}}-\frac{1}{2}\right) q+O\left(q^{7 / 8}\right)
$$

more factor base pairs. We want to end up using precisely all of the factor base pairs, of which there are $\left({ }_{2}^{\#} \mathcal{F}_{\chi}\right)=8 \eta^{8} q+O\left(q^{7 / 8}\right)$. We get the equation

$$
8 \eta^{8} q+O\left(q^{7 / 8}\right)=q \ln \left(\frac{\chi q^{1 / 4}}{4 \eta^{4}}\right)+\left(\frac{8 \eta^{4}}{\chi^{2}}-\frac{1}{2}\right) q+O\left(q^{7 / 8}\right) .
$$

Exponentiating this yields

$$
2 \eta^{2} \exp \left(4 \eta^{8}-4 \eta^{4} / \chi^{2}+1 / 4\right)=\chi^{1 / 2} q^{1 / 8}+O(1) .
$$

When $q$ is big we can approximate this with

$$
2 \eta^{2} \exp \left(4 \eta^{8}-4 \eta^{4} / \chi^{2}+1 / 4\right)=\chi^{1 / 2} q^{1 / 8}
$$

As in the proof of Theorem 1 we find that the average row weight is approximated from above by

$$
\begin{gathered}
16+\frac{2^{2}}{\# \mathcal{F}_{\chi}}\left[\sum_{k=N_{0}}^{\chi q^{3 / 4}-1}\left(1+\ln \left(\frac{k}{4 \eta^{4} q^{1 / 2}}\right)+O\left(q^{-3 / 8}\right)\right) \cdot\left(\frac{1}{2}+O\left(q^{-1 / 2}\right)\right) \frac{k / q}{1-k / q}\right. \\
\left.+\left(1+\ln \left(\frac{\chi q^{3 / 4}}{4 \eta^{4} q^{1 / 2}}\right)+O\left(q^{-3 / 8}\right)\right)\left(\left(4 \eta^{4}-\frac{\chi^{2}}{4}\right) q^{1 / 2}+O\left(q^{3 / 8}\right)\right)\right] \\
=16+\frac{\chi^{2} \ln q}{16 \eta^{4}}-\frac{\chi^{2} \ln \left(4 \eta^{4}\right)}{4 \eta^{4}}+\frac{\chi^{2}}{8 \eta^{4}}+\frac{\chi^{2} \ln \chi}{4 \eta^{4}} \\
+\left(1-\frac{\chi^{2}}{16 \eta^{4}}\right)\left(4-4 \ln \left(4 \eta^{4}\right)+\ln q+4 \ln \chi\right)+O\left(q^{-1 / 8} \ln q\right) \\
=20-\frac{\chi^{2}}{8 \eta^{4}}-4 \ln \left(4 \eta^{4}\right)+\ln q+4 \ln \chi+O\left(q^{-1 / 8} \ln q\right) .
\end{gathered}
$$

The computational complexity of the relation search step is given by the number of factor base pairs, which is roughly $8 \eta^{8} q$, multiplied by the computational cost of processing one such pair, as was discussed in the beginning of Section 4 The complexity of the linear algebra step depends quadratically on $\# \mathcal{F}_{\chi}$ and linearly on the average row weight given by Theorem 3 . The total memory cost is given by the size of the graph, which is $\chi q^{3 / 4}$, multiplied by the memory cost of storing one vertex. For a given $q$ the largest meaningful choice for $\chi$ is $\lambda$, given by Theorem 2 since at that point all of the required full relations have been found and there is nothing more to do. 
Remark 2. In fact, it is easy to see that $\eta$, as a function of $\chi$, has a global minimum at a point where $\chi=4 \eta^{2}$. Substituting this into (4) yields precisely the defining equation of $\lambda$.

On the other hand, the index calculus attack is no faster than Pollard rho unless $8 \eta^{8} q \ll q^{3 / 2}$, which yields the upper bound $\eta \ll 2^{-3 / 8} q^{1 / 16}$. We denote the $\chi$ corresponding to $\eta=2^{-3 / 8} q^{1 / 16}$ by $\chi_{\min }$. Hence we assume that the smallest meaningful size of the graph is $\chi_{\min } q^{3 / 4}$.

For any fixed $q$, the value of $\eta$ increases as $\chi$ decreases from $\lambda$ towards $\chi_{\min }$, but what is interesting is the rate of change of $\eta$. It turns out that $\eta$ increases quite slowly until $\chi$ gets close to 1 . In Figure 1 we show the ratios of both the relation search complexity and linear algebra complexity relative to those of Diem's algorithm, as functions of $\chi$, for several field sizes.

Fig. 1: Complexities relative to those of Diem's algorithm, for field sizes $q=2^{60}, 2^{80}, 2^{100}, 2^{120}, 2^{140}, 2^{160}, 2^{180}, 2^{200}$

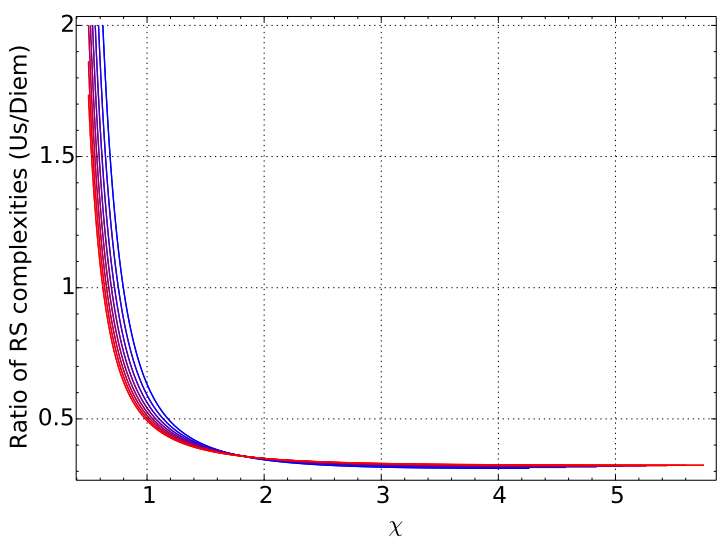

(a) Relation search $(\mathrm{RS})$

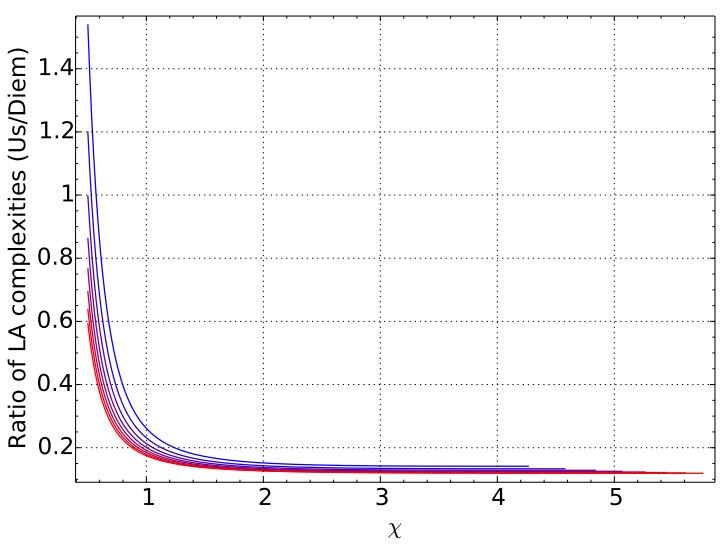

(b) Linear algebra (LA)

Another point of view is to look at the complexities of relation search and linear algebra relative to the best possible ones, i.e. those obtained in the case of unrestricted memory $\left(\chi=4 \lambda^{2}\right)$, as functions of memory cost relative to $N_{\max }$. These are presented in Figure2, again for several field sizes. These graphs are instructive since we already have presented the values for the fastest case $(\chi=\lambda)$ in Tables 1 and 3 . For example, consider the field size $q=2^{60}$ and $\chi /\left(4 \lambda^{2}\right)=1 / 5$. Then $(\eta / \lambda)^{8} \approx 2.75$, so the running time is 2.75 times that of the running time in the unrestricted case, but the memory use has been cut down to a fifth of the original. We get more concrete numbers by scaling the results of Table 1. The memory use is $10098 \mathrm{~TB}$ and the computational complexity is approximately $q^{1.226}=2^{73.589}$ field multiplications.

Yet a third point of view is to compare our algorithm to Pollard rho when memory use is even more restricted. Figure 3 shows the ratio of the complexities for several field sizes as a function of $\chi$. The key point to observe is that one can do significantly better than Pollard rho even if $\chi$ is taken to be very small, so if one has massive amounts of computing power available the best approach might be to choose some suitable $\chi \ll 1$, although the memory cost is still going to be extremely large. For example, if we take $q=2^{70}$ and $\chi=2^{-6} \approx 0.016$, the graph will end up containing $\chi q^{3 / 4}=2^{46.5} \approx 10^{14.0}$ vertices. Using the same estimate as in Section 4 , this corresponds to $67300 \mathrm{~TB}$ of memory. The complexity of relation search will be $8 \eta^{8} q \approx q^{1.386}$. As in Section 4. we can use the value $7 \log _{2} q+13$ for the number of field multiplications needed to process one pair of factor base elements, so the total number of field multiplications can be estimated to be $\left(7 \log _{2} q+13\right) \cdot 8 \eta^{8} q \approx q^{1.514}$. Of course this is bigger than $q^{3 / 2}$, but the unit of time in Pollard rho and other generic algorithms involves operations in the Jacobian, and for non-hyperelliptic genus 3 curves each group operation costs around 130-185 field multiplications plus 2 field inversions FOR.

Recall that in Diem's algorithm (see [Die2]) the size of the factor base should be taken to be at least

$$
\# \mathcal{F}_{\text {Diem }}:=\left\lceil((3 / 2) \ln q+4)^{1 / 2} q^{1 / 2}\right\rceil
$$


Fig. 2: Complexities relative to the case of unrestricted memory, for field sizes

$$
q=2^{60}, 2^{80}, 2^{100}, 2^{120}, 2^{140}, 2^{160}, 2^{180}, 2^{200}
$$

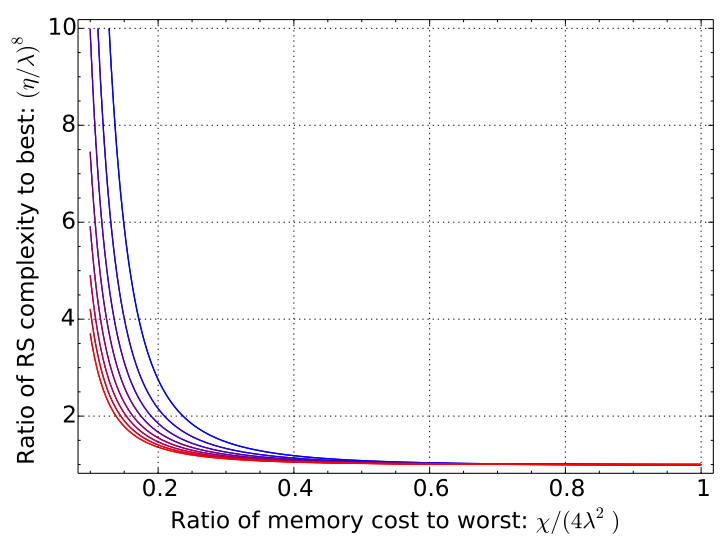

(a) Relation search (RS)

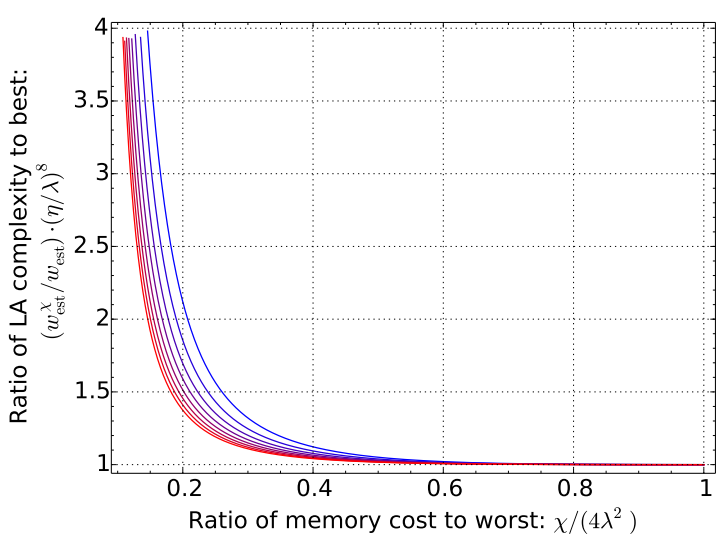

(b) Linear algebra (LA)

Fig. 3: Relation search complexity relative to Pollard rho, for field sizes $q=2^{60}, 2^{65}, 2^{70}, 2^{75}, 2^{80}, 2^{85}, 2^{90}, 2^{95}, 2^{100}$

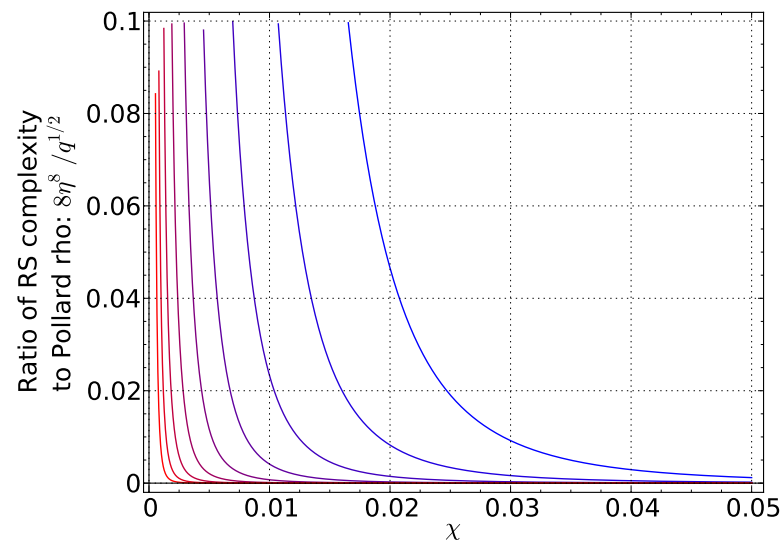

whereas the size of our factor base is $\# \mathcal{F}_{\chi}=4 \eta^{4} q^{1 / 2}$. Using the defining equation of $\eta$ to write

$$
\ln q=32 \eta^{8}-\frac{32 \eta^{4}}{\chi^{2}}+2+8 \ln \left(2 \eta^{2}\right)-4 \ln \chi
$$

we find that

$$
\rho_{\mathrm{RS}}^{\chi}(q):=\left(\frac{\# \mathcal{F}_{\chi}}{\# \mathcal{F}_{\text {Diem }}}\right)^{2}=\frac{1}{3}\left(1-\frac{1}{\eta^{4} \chi^{2}}+\frac{1}{16 \eta^{8}}+\frac{\ln \left(2 \eta^{2}\right)}{4 \eta^{8}}-\frac{\ln \chi}{8 \eta^{8}}+\frac{1}{24 \eta^{8}}\right)^{-1}
$$

which approaches $1 / 3$ as $q \rightarrow \infty$ no matter what the fixed number $\chi$ is. This is the ratio plotted in Figure 1 on the left-hand side. Note that both in Diem's algorithm and in our variant the size of the factor base is chosen to be minimal so that all pairs of factor base elements are being used, so if we assume that in both algorithms the complexity of processing a pair of factor base elements is the same, the ratio $\rho_{\mathrm{RS}}^{\chi}$ measures the ratio of the complexities of the relation search steps. 
Similarly, we define

$$
\rho_{\text {LA }}^{\chi}(q):=\frac{20-\chi^{2} /\left(8 \eta^{4}\right)-4 \ln \left(4 \eta^{4}\right)+\ln q+4 \ln \chi}{6+3 \ln q}\left(\frac{\# \mathcal{F}_{\chi}}{\# \mathcal{F}_{\text {Diem }}}\right)^{2} .
$$

The ratio $\rho_{\mathrm{LA}}^{\chi}$ approaches $1 / 9$ as $q \rightarrow \infty$ and it is the ratio plotted in Figure 1 on the right-hand side.

Lemma 2. For any fixed $\chi$, asymptotically as $q \rightarrow \infty$ our algorithm is 3 times faster than Diem's algorithm for relation search and 9 times faster for linear algebra.

Remark 3. As we have pointed out earlier, in our algorithm it is possible to take the factor base to be slightly smaller and re-run the graph building/relation searching step once it has failed for the first time. At that point the graph is huge and it should not take long to find the missing full relations. This is not possible in Diem's approach. We also save time because we only need to find roughly $q^{1 / 8}$ random points on the curve compared to over $q^{1 / 2}$ which have to be found in Diem's algorithm. Of course we then need to compute more intersection divisors to find the remaining factor base elements, but all of that work also builds the graph.

Remark 4. There is another variant of Diem's algorithm, namely the full algorithm approach of Diem and Thomé [DT] (see GTTD] for the hyperelliptic case). In this algorithm a very large graph is constructed, of the size $q^{5 / 6}$. Consequently the factor base can be chosen to be smaller, of the size $2 q^{1 / 2}$. The graph is disconnected and is searched for cycles which are then used to produce full relations. The complexity analysis of the full algorithm is difficult and the large graph size makes is impractical for all but the smallest examples so we will not discuss it further here.

\section{Parallelization}

In this section we study how the work of computing the DLP can be split between $K$ computers. It turns out that with an increase in total computing time and total memory cost we can be split the computation between these $K$ computers so that each needs to pay only a fraction of the original memory cost.

Suppose $\lambda_{K}$ is a real number and we start by choosing a set $\mathcal{R} \mathcal{P}^{K}$ of $4 \lambda_{K} q^{1 / 8}$ random points on the curve and as usual use these to generate a factor base $\mathcal{F}^{K}$ of size $4 \lambda_{K}^{4} q^{1 / 2}$. As usual at this point the graph will contain $4 \lambda_{K}^{4} q^{1 / 2}$ triangles. Now distribute the entire factor base and the base vertices to each one of the $K$ computers and split the triangles evenly between them so that each computer gets $4 \lambda_{K}^{4} q^{1 / 2} / K$ triangles. Each computer starts building a graph and searching for full relations using their share of the triangles until they have each found approximately $4 \lambda_{K}^{4} q^{1 / 2}$ full relations. Now all full relations are combined into a matrix and the linear algebra step is performed as usual. To simplify the notation, we denote $\lambda_{K}$ simply by $\lambda$.

Theorem 4. (Heuristic) If $q$ is large enough and we take $\lambda$ to be a root of

$$
\lambda \exp \left(4 \lambda^{8}\right)=\left(K^{2} q\right)^{1 / 8}
$$

we can expect the algorithm to terminate successfully. Each of the $K$ computers will end up with a graph of size

$$
N_{\max }=\frac{4 \lambda^{2} q^{3 / 4}}{\sqrt{K}}
$$

The average row weight of the matrix will be approximately

$$
18-8 \ln \lambda+\ln \left(K^{2} q\right) .
$$

Proof. The proof is again similar to the proof of Theorem 2 We start with a set $\mathcal{R} \mathcal{P}^{K}$ of $4 \lambda q^{1 / 8}=O\left(q^{1 / 8}\right)$ random points and suppose that $N_{\max }=O\left(q^{3 / 4}\right)$. The size of the factor base and the number of vertices in the graph after the triangles have been constructed are

$$
\# \mathcal{F}^{K}=4 \lambda^{4} q^{1 / 2}+O\left(q^{3 / 8}\right), \quad N_{0}=4 \lambda^{4} q^{1 / 2}+O\left(q^{3 / 8}\right) .
$$

Building the graphs from size $N_{0} / K$ to $N_{\max }$ on each computer produces

$$
\left(\frac{1}{2}+O\left(q^{-1 / 2}\right)\right) \sum_{k=N_{0} / K}^{N_{\max }-1} \frac{k / q}{1-k / q}=\frac{N_{\max }^{2}}{4 q}+O\left(q^{1 / 4}\right)
$$


full relations. But each computer should produce $\# \mathcal{F}^{K} / K=4 \lambda^{4} q^{1 / 2} / K+O\left(q^{3 / 8}\right)$ full relations, so we get an equation and solve

$$
N_{\max }=\frac{4 \lambda^{2} q^{3 / 4}}{\sqrt{K}}\left(1+O\left(q^{-1 / 8}\right)\right)=\frac{4 \lambda^{2} q^{3 / 4}}{\sqrt{K}}+O\left(q^{5 / 8}\right) .
$$

The number of pairs of factor base elements that each computer has is $\left(\begin{array}{c}\mathcal{F}^{K} \\ 2\end{array}\right)=8 \lambda^{8} q+O\left(q^{7 / 8}\right)$. We want this number to equal the number of pairs needed to build the graphs as explained above. Hence we need

$$
8 \lambda^{8} q+O\left(q^{7 / 8}\right)=\left(1+O\left(q^{-1 / 2}\right)\right) \sum_{k=N_{0} / K}^{N_{\max }-1} \frac{1}{(k / q)(1-k / q)}=q \ln \left(\frac{K^{1 / 2} q^{1 / 4}}{\lambda^{2}}\right)+O\left(q^{7 / 8}\right)
$$

which gives the equation

$$
\lambda \exp \left(4 \lambda^{8}\right)=\left(K^{2} q\right)^{1 / 8}+O(1)
$$

Finally, the average row weight is approximated from above by

$$
\begin{gathered}
16+\frac{2^{2}}{\# \mathcal{F}^{K} / K} \sum_{k=N_{0} / K}^{N_{\max }-1}\left(1+\ln \left(\frac{k K}{4 \lambda^{4} q^{1 / 2}}\right)+O\left(q^{-3 / 8}\right)\right) \cdot\left(\frac{1}{2}+O\left(q^{-1 / 2}\right)\right) \frac{k / q}{1-k / q} \\
=18-8 \ln \lambda+\ln \left(K^{2} q\right)+O\left(q^{-1 / 8} \ln q\right) .
\end{gathered}
$$

More generally, we can consider what happens if each of the $K$ computers stops building their graphs when they reach size $\chi q^{3 / 4} / \sqrt{K}$, where $\chi$ is a fixed positive real number, and only proceed with relation search. Suppose $\eta_{K, \chi}$ is a real number and we start by choosing a set $\mathcal{R} \mathcal{P}_{\chi}^{K}$ of $4 \eta_{K, \chi} q^{1 / 8}$ random points on the curve. Then we get a result similar to Theorem 3 To simplify the notation, we denote $\eta_{K, \chi}$ by $\eta$.

Theorem 5. (Heuristic) If $q$ is large enough and we take $\eta$ to be a root of

$$
2 \eta^{2} \exp \left(4 \eta^{8}-4 \eta^{4} / \chi^{2}+1 / 4\right)=\chi^{1 / 2}\left(K^{2} q\right)^{1 / 8}
$$

we can expect the algorithm to terminate successfully. The average row weight of the matrix will be approximately

$$
20-\frac{\chi^{2}}{8 \eta^{4}}-4 \ln \left(4 \eta^{4}\right)+\ln \left(K^{2} q\right)+4 \ln \chi
$$

Proof. Similar to the proofs of Theorem 3 and Theorem 4

Above we assumed that each one of the computers computes the same $8 \eta^{8} q$ intersection divisors but of course this is completely unnecessary if there is a very efficient way for the computers to communicate with each other. In this case each one computes $\left(1 / 2+O\left(q^{-1 / 2}\right)\right) 8 \eta^{8} q / K$ intersection divisors and shares their results with the other $K-1$ computers. Unfortunately this is a massive amount of data to share. In fact, merely storing all these intersection divisors costs potentially much more memory than storing the graph since

$$
\frac{4 \eta^{8} q}{K} \gg \frac{\chi q^{3 / 4}}{\sqrt{K}}
$$

for practical field sizes unless $K$ is impossibly large. Hence instead of storing the intersection divisors they should be streamed to all other computers as soon as they are generated and then deleted immediately afterwards. This speeds up the algorithm only if the connections between the computers are so fast that distributing the data over the network is faster than for each computer to generate it separately. We assume this is the case.

Let $M_{\text {Total }}^{K, \chi}$ be the total number of field multiplications needed per computer. We have

$$
M_{\text {Total }}^{K, \chi}=M_{\text {pair }} \cdot \frac{1}{K}\left(\begin{array}{c}
\# \mathcal{F}_{\chi}^{K} \\
2
\end{array}\right) \approx\left(7 \log _{2} q+13\right) \cdot \frac{8 \eta_{K, \chi}^{8} q}{K}
$$

Note that this is not exactly the same as $1 / K$-th of the complexity in the unparallelized case because the coefficient $\eta_{K, \chi}$ is bigger, namely $\eta_{K, \chi}(q)=\eta_{\chi}\left(K^{2} q\right)$. 
The complexity of linear algebra is given by

$$
\left(20-\frac{\chi^{2}}{8 \eta_{K, \chi}^{4}}-4 \ln \left(4 \eta_{K, \chi}^{4}\right)+\ln \left(K^{2} q\right)+4 \ln \chi\right) \cdot 16 \eta_{K, \chi}^{8} q,
$$

which is worse than the complexity in the unparallelized case, again due to $\eta_{K, \chi}(q)=\eta_{\chi}\left(K^{2} q\right)$. We demonstrate these results in the case of unrestricted memory, i.e. when $\chi=4 \lambda_{K}^{2}$. The ratio of complexities of the linear algebra steps in the parallelized and unparallelized cases is

$$
\rho_{\mathrm{LA}}^{K}:=\frac{18-8 \ln \lambda_{K}+\ln \left(K^{2} q\right)}{18-8 \ln \lambda+\ln q}\left(\frac{\lambda_{K}}{\lambda}\right)^{8} .
$$

We denote

$$
\mathrm{MPC}_{K}(\text { Memory cost Per Computer }):=\frac{4 \lambda_{K}^{2} q^{3 / 4}}{\sqrt{K}} \cdot\left(\left\lceil\left(\log _{2} q\right) / 64\right\rceil \cdot 370 \text { bytes }\right)
$$

and

$$
\mathrm{IDPC}_{K} \text { (Intersection Divisors Per Computer with } \mathbb{F}_{q} \text {-rational points) }:=\frac{4 \lambda_{K}^{8} q}{K},
$$

which measures the amount of data that needs to be shared.

These numbers for a few practical field sizes are shown in Tables 4 5 6 It is also easy to see how to scale the values in Tables 4, 5, 6 to obtain correspondings numbers in the case $\chi<4 \lambda_{K}^{2}$. For example, to find $M_{\text {Total }}^{K, \chi}$, simply scale the value of $M_{\text {Total }}^{K}$ by the coefficient given by Figure 2 for a field of size $K^{2} q$. To find $\mathrm{MPC}_{K, \chi}$, scale the value of $\mathrm{MPC}_{K}$ by $\left(\eta_{K, \chi} / \lambda_{K}\right)^{2}$. Concretely, consider e.g. $q=2^{60}, K=100$ and $\chi /\left(4 \lambda_{K}^{2}\right)=1 / 50$. Then

$$
\log _{2} M_{\text {Total }}^{K, \chi}=\log _{2}\left[M_{\text {Total }}^{K} \cdot\left(\frac{\eta_{K, \chi}}{\lambda_{K}}\right)^{8}\right]=79.03607
$$

and $\mathrm{MPC}_{K, \chi}=106 \mathrm{~TB}$.

In the above our main concern was with reducing the per computer memory cost. One should keep in mind that parallelization makes the complexity of the linear algebra part slightly worse and for large $K$ the difference between the relation search step and the linear algebra step becomes very large, unless $\chi$ is taken to be smaller. To make linear algebra faster, each computer could perform some preprocessing of the full relations before they are combined into the full linear algebra problem. There are also several ways of parallelizing relation search within the $K$ memory units to further reduce the complexity $M_{\text {Total. }}^{K, \chi}$. For instance, each processor connected to a memory unit can compute its own share of intersection divisors which are then collected together and used to build the local graph. 
Table 4: Parallelization: $K=4$

\begin{tabular}{c|c|c|c|c} 
Field size $q$ & $\log _{2} M_{\text {Total }}$ & $\rho_{\text {LA }}$ & MPC & $\log _{2}$ IDPC \\
\hline $2^{60}$ & 70.21903 & 1.11420 & $25648 \mathrm{~TB}$ & 60.46080 \\
$2^{70}$ & 80.64203 & 1.09991 & $9.62 \cdot 10^{6} \mathrm{~TB}$ & 70.66761 \\
$2^{80}$ & 91.01129 & 1.08883 & $1.80 \cdot 10^{9} \mathrm{~TB}$ & 80.84890 \\
$2^{90}$ & 101.3389 & 1.07996 & $3.35 \cdot 10^{11} \mathrm{~TB}$ & 91.01023 \\
$2^{100}$ & 111.63331 & 1.07272 & $6.21 \cdot 10^{13} \mathrm{~TB}$ & 101.15555 \\
$2^{110}$ & 121.90060 & 1.06667 & $1.15 \cdot 10^{16} \mathrm{~TB}$ & 111.28773 \\
$2^{120}$ & 132.14535 & 1.06156 & $2.13 \cdot 10^{18} \mathrm{~TB}$ & 121.40894 \\
$2^{140}$ & 152.58044 & 1.05338 & $1.09 \cdot 10^{23} \mathrm{~TB}$ & 141.62479 \\
$2^{160}$ & 172.95868 & 1.04712 & $3.67 \cdot 10^{27} \mathrm{~TB}$ & 161.81275 \\
$2^{180}$ & 193.29321 & 1.04217 & $1.24 \cdot 10^{32} \mathrm{~TB}$ & 181.97919 \\
$2^{200}$ & 213.59307 & 1.03816 & $5.56 \cdot 10^{36} \mathrm{~TB}$ & 202.12853
\end{tabular}

Table 5: Parallelization: $K=100$

\begin{tabular}{|c|c|c|c|c|}
\hline ield size & $\log _{2} M_{\text {Total }}$ & $\rho_{\mathrm{LA}}$ & MPC & $\log _{2}$ IDPC \\
\hline $2^{60}$ & 65.76817 & 1.40309 & $5304 \mathrm{~TB}$ & 56.00995 \\
\hline & 76.16726 & 1.35025 & $1.98 \cdot 10^{6} \mathrm{~TB}$ & 66.19284 \\
\hline $2^{80}$ & 86.51785 & 1.30967 & $3.69 \cdot 10^{8} \mathrm{~TB}$ & 76.35545 \\
\hline $2^{90}$ & 96.83049 & 1.27752 & $6.85 \cdot 10^{10} \mathrm{~TB}$ & 86.50181 \\
\hline & 107.11261 & 1.25142 & $1.27 \cdot 10^{13} \mathrm{~TB}$ & 96.63485 \\
\hline 110 & 117.36965 & 1.22982 & $2.35 \cdot 10^{15} \mathrm{~TB}$ & 106.75678 \\
\hline $2^{120}$ & 127.60571 & 1.21162 & $4.33 \cdot 10^{17} \mathrm{~TB}$ & 116.86931 \\
\hline & 148.0 & 1.1827 & $2.20 \cdot 10^{22} \mathrm{~TB}$ & 137.07124 \\
\hline $2^{160}$ & 168.39449 & 1.16072 & $7.45 \cdot 10^{26} \mathrm{~TB}$ & 157.24856 \\
\hline $2^{180}$ & 188.72061 & 1.14346 & $2.51 \cdot 10^{31} \mathrm{~TB}$ & 177.40660 \\
\hline & 209.01367 & 1.12954 & $1.12 \cdot 10^{36} \mathrm{~TB}$ & 197.54912 \\
\hline
\end{tabular}

Table 6: Parallelization: $K=1000$

\begin{tabular}{c|c|c|c|c} 
Field size $q$ & $\log _{2} M_{\text {Total }}$ & $\rho_{\text {LA }}$ & MPC & $\log _{2}$ IDPC \\
\hline $2^{60}$ & 62.57026 & 1.63014 & $1714 \mathrm{~TB}$ & 52.81204 \\
$2^{70}$ & 72.95535 & 1.54507 & $6.39 \cdot 10^{5} \mathrm{~TB}$ & 62.98094 \\
$2^{80}$ & 83.29477 & 1.48017 & $1.19 \cdot 10^{8} \mathrm{~TB}$ & 73.13238 \\
$2^{90}$ & 93.59828 & 1.42905 & $2.20 \cdot 10^{10} \mathrm{~TB}$ & 83.26961 \\
$2^{100}$ & 103.87281 & 1.38774 & $4.07 \cdot 10^{12} \mathrm{~TB}$ & 93.39505 \\
$2^{110}$ & 114.12344 & 1.35366 & $7.52 \cdot 10^{14} \mathrm{~TB}$ & 103.51057 \\
$2^{120}$ & 124.35401 & 1.32508 & $1.39 \cdot 10^{17} \mathrm{~TB}$ & 113.61761 \\
$2^{140}$ & 144.76630 & 1.27981 & $7.05 \cdot 10^{21} \mathrm{~TB}$ & 133.81065 \\
$2^{160}$ & 165.12699 & 1.24559 & $2.38 \cdot 10^{26} \mathrm{~TB}$ & 153.98106 \\
$2^{180}$ & 185.44760 & 1.21881 & $8.00 \cdot 10^{30} \mathrm{~TB}$ & 174.13358 \\
$2^{200}$ & 205.73615 & 1.19728 & $3.58 \cdot 10^{35} \mathrm{~TB}$ & 194.27161
\end{tabular}




\section{References}

[ADH] L. M. Adleman, J. DeMarrais, M.-D. Huang, A subexponential algorithm for discrete logarithms over hyperelliptic curves of large genus over $G F(q)$, Theoret. Comput. Sci. 226 (1999), no. 1-2, pp. 7-18.

[BCLS] D.J. Bernstein, C. Chuengsatiansup, T. Lange, P. Schwabe, Kummer strikes back: new DH speed records, http://cr.yp.to/papers.html\#kummer, Preprint 2014, 21pp.

[BCHL] J. W. Bos, C. Costello, H. Hisil, K. Lauter, Fast Cryptography in Genus 2, Advances in Cryptology - EUROCRYPT 2013, Lecture Notes in Computer Science, May 2013.

[DT] Diem, C., Thomé, E., Index Calculus in Class Groups of Non-Hyperelliptic Curves of Genus Three, Journal of Cryptology 21 (2008), no. 4, 591-611.

[Die] Diem, C., An Index Calculus Algorithm for Plane Curves of Small Degree, Algorithmic number theory, 543-557, Springer Berlin Heidelberg, 2006.

[Die2] Diem, C., Index Calculus in Class Groups of Non-Hyperelliptic Curves of Genus 3 from a Full Cost Perspective, http://www.math.uni-leipzig.de/〜diem/preprints/sharcs. pdf.

[Eng] Enge, A., Computing discrete logarithms in high-genus hyperelliptic jacobians in provably subexponential time, Math. Comp. 71 (2002), no. 238, pp. 729-742.

[EG] Enge, A., Gaudry, P., A general framework for subexponential discrete logarithm algorithms, Acta Arith. 102 (2002), no. 1, pp. 83-103.

[FOR] Flon, S., Oyono, R., Ritzenthaler, C., Fast addition on non-hyperelliptic genus 3 curves, IACR Cryptology ePrint Archive 2004 (2004): 118.

[Gau] Gaudry, P., An algorithm for solving the discrete log problem on hyperelliptic curves, Advances in Cryptology - EUROCRYPT 2000, Springer-Verlag, LNCS 1807, pp. 19-34, 2000.

[GTTD] Gaudry, P., Thomé , E., Thériault, N., Diem, C., A Double Large Prime Variation for Small Genus Hyperelliptic Index Calculus, Math. Comp. 76 (2007), no. 257, 475-492.

[Hes] Hess, F., Computing Riemann-Roch spaces in algebraic function fields and related topics, J. Symbolic Comput. 33 (2002), no. 4, 425-445.

[Kob] Koblitz, N., Hyperelliptic Cryptosystems, Journal of Cryptology 1 (1989), 139-150.

[Nag] Nagao, K., Index calculus for Jacobian of hyperelliptic curve of small genus using two large primes, Japan Journal of Industrial and Applied Mathematics, 24, no.3 (2007).

[Smi] Smith, B., Isogenies and the Discrete Logarithm Problem in Jacobians of Genus 3 Hyperelliptic Curves, Advances in Cryptology - EUROCRYPT 2008, 163-180, Springer Berlin Heidelberg, 2008.

[The] Thériault, N., Index calculus attack for hyperelliptic curves of small genus, Advances in Cryptology - ASIACRYPT 2003, New York: Springer, 2003.

[VJS] M. D. Velichka, M. J. Jacobson Jr., A. Stein, Computing discrete logarithms in the Jacobian of high-genus hyperelliptic curves over even characteristic finite fields Math. Comp. 83 (2014), 935-963. 